\title{
NEUROSCIENCE
}

\section{Molecular matchmaking for neural control}

Engineered ion channels and cognate synthetic ligands expand the catalog of tools to study brain function.

Behind every beautiful mind operates a molecular clockwork of concerted ion and small-molecule fluxes. The discovery that some light-gated ion channels and pumps of microbes can be 'transplanted' to control ionic conductance in the mammalian brain has provided a unique technology, dubbed optogenetics, to study neural circuitry with respect to animal physiology and behavior. Nevertheless, these approaches have only recently established a budding branch of modern neuroscience, and researchers continue to seek optimized molecular switches for neuron silencing or activation.

Rather than focus on the exotic tools of optogenetics, Scott Sternson and his colleagues at Janelia Farm undertook a different strategy toward genetically encoded neural control. The researchers recently presented a highly customizable toolbox of engineered ion channels with a modular design: a ligand-sensing domain derived from a natural receptor for the neurotransmitter acetylcholine (ACh) can be transplanted onto the ion-conductive pore of a plethora of related channels. Expressed in targeted neurons, the resulting chimeras confer specificity for ionic fluxes in response to tailored exogenous ligands.

Channels from the ACh receptor family are a well-trodden ground for neuroscientists. "We have the capability to leverage an extremely rich literature of structurefunction studies on the properties of these receptors," explains Sternson. “That gives us a huge amount of control over conductance properties-whether it is desensitization, magnitude of conductance or ion selectivity-based on the forty years of work that have preceded ours."

Tapping into this wealth of scientific wisdom, Sternson and colleagues not only developed highly selective channel-ligand pairs but also ensured their suitability for applications in vivo. This required both the protein actuators to be insensitive to endogenous neurotransmitters and the matching ligands to be 'blind' for natural receptors while penetrating deep into the live brain with minimal side effects on physiology.
The researchers started with a homologybased structural model of the ACh receptor bound to a synthetic agonist known to affect neuronal function in vivo. Pairing rational protein mutagenesis with chemical synthesis and functional assays, the researchers screened more than 1,000 ligand-channel combinations. Several of these interacted tightly and were also selective against each other or their natural counterparts. Stitching the ligand-binding modules onto different ion-pore domains preserved the selectivity of ligand sensing and ion flux activation.

The researchers tested several chimeric channels to modulate neuron activation, calcium flux or silencing in isolated brain tissues from mice. By applying cognate ligands, they could effectively puppeteer neuronal function. The engineered proteins proved to be especially attractive for silencing applications, as they decreased neuronal excitability "through a shunting mechanism that can often be more effective than the hyperpolarization mechanism of current optogenetic silencing tools," explains Sternson.

Nevertheless, both light- and ligandsensitive tools have advantages and can be used in concert for sophisticated studies of neural circuitry. To demonstrate this in vivo, the authors expressed together lightand ligand-gated ion channels of opposing activity in hypothalamic neurons controlling hunger and related survival behaviors. Lightstimulation provided instantaneous neuron activation resulting in voracious appetite and overeating, whereas peripheral administration of inhibitory ligands effectively tamed this ravenous behavior.

Although currently the effects of peripheral ligand administration are relatively slow, Sternson believes that photoisomerizable or caged ligand versions will soon enable experiments of high temporal precision. $\mathrm{He}$ is optimistic that multiple pharmacologically targeted components can be used in the same animal to explore the role of ion flux in neural function as well as other aspects of cell biology and animal physiology.

Petya V Krasteva

RESEARCH PAPERS

Magnus, C.J. et al. Chemical and genetic engineering of selective ion channel-ligand interactions. Science 333, 1292-1296 (2011). 